Original article

\title{
The high-fat diet induces myocardial fibrosis in the metabolically healthy obese minipigs - The role of ER stress and oxidative stress
}

\author{
Sin-Jin Li ${ }^{a}$, Chia-Hsin Liu a , Hsien-Pin Chu ${ }^{b}$, Harry J. Mersmann ${ }^{a}$, Shih-Torng Ding a , \\ Chun-Han Chu ${ }^{a}$, Chia-Yu Wang a, Ching-Yi Chen ${ }^{\text {a, ** }}$ \\ a Department of Animal Science and Technology, National Taiwan University, No. 50, Lane 155, Sec 3, Keelung Rd, Taipei, 10672, Taiwan \\ b Taitung Animal Propagation Station, Livestock Research Institute Council of Agriculture, No. 30, Binlang Vil., Beinan Township, Taitung County, 95444, \\ Taiwan
}

\section{A R T I C L E I N F O}

\section{Article history:}

Received 29 October 2015

Accepted 1 June 2016

\section{Keywords:}

Metabolic syndrome

Metabolically healthy obesity

Autophagy

ER stress

Oxidative stress

Cardiomyopathy

\begin{abstract}
S U M M A R Y
Background: The cellular mechanisms of obesity-induced cardiomyopathy are multiple and not completely elucidated. The objective of this study was to differentiate two obesity-associated cardiomyopathy miniature pig models: one with the metabolic syndrome (MetS), and one with a metabolically healthy obesity (MHO). The cellular responses during the development of obesity-induced cardiomyopathy were investigated.

Methods: Five-month-old Lee-Sung (MetS) and Lanyu (MHO) minipigs were made obese by feeding a high-fat diet (HFD) for 6 months.

Results: Obese pigs exhibited a greater heart weight than control pigs. Interstitial and perivascular fibrosis developed in the myocardium of obese pigs. The HFD induced cardiac lipid accumulation and oxidative stress and also decreased the antioxidant defense in MetS pigs. This diet activated oxidative stress without changing cardiac antioxidant defense and lipid content in MHO pigs. The HFD upregulated the expression of Grp94, CHOP, caspase 12, p62, and LC3II, and increased the ratio of LC3II to LC3I in the left ventricle (LV) of MetS pigs. Compared to obese MetS pigs, less Grp94 and elevated CHOP expression was found in the obese MHO heart. The HFD did not change the ratio of LC3II to LC3I and p62 expression in obese MHO pigs. The obese MetS pigs had an extensive and greater inflammatory response in the plasma than the obese MHO pigs, which had a lesser and milder inflammation.

Conclusion: Oxidative stress and ER stress were involved in the progression of MHO-related cardiomyopathy. Inflammation, autophagy, ER stress, oxidative stress, and lipotoxicity participated in the pathological mechanism of MetS-related cardiomyopathy.
\end{abstract}

๑) 2016 Elsevier Ltd and European Society for Clinical Nutrition and Metabolism. All rights reserved.

\section{Introduction}

Obesity is considered the main contributing factor to the development of the metabolic syndrome (MetS) and metabolic related diseases [1-3]; however, not all obese individuals exhibit cardio-metabolic abnormalities. Recent studies identify a subset of obese individuals, the metabolically healthy obesity (MHO) who have a low burden of adiposity-related metabolic abnormalities compared to obese individuals with MetS [4]. Depending on the

\footnotetext{
* Corresponding author. Present address: Department of Animal Science and Technology, National Taiwan University, 50, Lane 155, Sec 3, Keelung Rd, Taipei, 10672, Taiwan. Tel.: +886 2 33665283; fax: +886 227324070.

E-mail address: ronichen@ntu.edu.tw (C.-Y. Chen).
}

definition used for cardio-metabolic markers, the prevalence of MHO varies from 12 to $43 \%$ in cohort studies [4-6]. Compared to obese subjects with MetS, several studies conclude that MHO individuals have reduced risks of cardiovascular diseases (CVDs) and stroke [5], acute myocardial infarction [7] and type II diabetes mellitus (T2DM) [6]. The MHO individuals have an increased risk of heart failure, particularly if they have long-lasting or severe obesity [7].

Obesity has been regarded as an independent risk factor and a direct cause of CVDs [2]. Severe obesity causes hemodynamic alterations, induces structural and functional changes in the heart, and prolonged severe obesity eventually leads to the development of heart failure [8]. The cellular mechanisms of MetS-related cardiomyopathy are multiple, and have been studied extensively, including lipotoxicity, cardiac hypertrophy and inflammation [8]. 
The protein quality control system, including the endoplasmic reticulum (ER) and autophagy, is responsible for the degradation of damaged proteins and organelles inside the cells, and plays a critical role in MetS-related cardiac injury [9]. In our previous mouse studies, cardiac injury with metabolic dysregulation was associated with autophagy dysregulation and ER stress $[9,10]$. A cell model suggests a pro-survival role of autophagy in cardiomyocyte apoptosis after exposure to lipotoxicity [10].

The underlying mechanism involved in MetS-related heart failure has been studied recently, whereas the mechanism for MHO-related heart failure is still unknown due to the difficulty to obtain human samples and lack of an optimal animal model. We previously established two diet-induced obesity minipig models one MetS (obesity with hyperglycemia, dyslipidemia and hypertension) and one MHO (obesity without any metabolic dysfunction), and found that AMPK and SIRT1 are involved in the development of MetS-induced fatty liver [11]. The current study showed cardiac hypertrophy and fibrosis in both obese MetS and MHO pigs. Accordingly, these two pig models were used to explore the relationship between obesity-induced cardiomyopathy and the intercellular protein quality control system. In addition, plasma cytokine profiles were investigated to differentiate the inflammatory response between obese MetS- and MHO-pigs.

\section{Materials and methods}

\subsection{Animals and experiment diets}

The investigation conforms to the Guide for the Care and Use of Laboratory Animals published by the US National Institutes of Health (NIH Publication No. 85-23, revised 1985). All animal care procedures used in this study were approved by the Institutional Animal Care and Use Committee of the National Taiwan University. Lanyu minipigs (as MHO model) from the Taitung Animal Propagation Station (Taitung, Taiwan) and Lee-Sung (as MetS model) pigs from National Taiwan University were used in this study. The obese pig model and feed formulations were established according to the previous study [11]. Five-month-old Lanyu (body weight: $18.3 \pm 1.5 \mathrm{~kg}$ ) and Lee-Sung miniature pigs (body weight: $18.1 \pm 0.8 \mathrm{~kg}$ ) were each randomly divided into two groups and fed either a C, control diet $(2436 \mathrm{Kcal} / \mathrm{kg}$ metabolic energy, consisting of $35.5 \%$ corn, $8 \%$ soybean meal, $15.6 \%$ soybean hulls, $20.7 \%$ alfalfa meal, $16 \%$ wheat bran, $3 \%$ molasses, $0.2 \%$ methionine, $0.1 \%$ lysine, $0.5 \% \mathrm{NaCl}, 0.2 \%$ choline and $0.3 \%$ vitamin and mineral premix) or a HFD, high fat diet (3786 Kcal/kg metabolic energy, consisting of $42.7 \%$ corn, $14 \%$ soybean meal, $15 \%$ lard and 20\% sucrose, $3 \%$ molasses, $0.3 \%$ methionine, $0.3 \%$ lysine, $3.2 \% \mathrm{CaHPO}_{4}, 0.6 \% \mathrm{CaCO}_{3}$, $0.5 \% \mathrm{NaCl}, 0.2 \%$ choline and $0.3 \%$ vitamin and mineral premix) for 6 months. Each treatment included 5 castrated male and 5 female minipigs; pigs of the same treatment were housed together in a sheltered pen. Feed was provided twice daily [11], and water was available ad libitum. BMI was measured as body weight/ (crown-rump length) ${ }^{2}$ [12].

At the end of the experiment, blood samples were taken from the jugular vein after an over-night fast. Animals were then sacrificed by intravenous injection of barbiturate in overdose and the left ventricles (LV) were excised, frozen in liquid nitrogen and stored at $-80^{\circ} \mathrm{C}$ until analysis.

\subsection{Histological and Mason trichrome staining}

Fresh LV samples $\left(1 \mathrm{~cm}^{3}\right)$ were fixed in $10 \%$ neutral formalin solution for one week, embedded in paraffin and sectioned at $5 \mu \mathrm{m}$. The sections were stained with Masson's trichrome stain for cardiac fibrosis determination. All stained LV sections were analyzed by Image J software [11].

\subsection{Measurement of total collagen content}

Sirius red is a unique dye which specifically binds to the [Gly-XY]n helical structure in collagen, and was used to quantify the degree of cardiac fibrosis in this study. The collagen was quantified with the Sirius Red Collagen Detection kit (Chondrex, Redmond, WA, USA) following the standard protocols provided with the assay kit. Homogenized LV tissues were incubated at $4{ }^{\circ} \mathrm{C}$ for $48 \mathrm{~h}$ in an acid-pepsin solution. The supernate was collected in tubes pretreated with buffered fetal bovine serum. Sirius Red solution was added to the supernate, then mixed and centrifuged. A calibration curve was obtained using the collagen standard in the assay kit and the absorbance was measured at $530 \mathrm{~nm}$ using microplate assays (BioTek, Synergy H1, Ashton Vale, BRS, USA).

\subsection{Triglyceride content and oxygen radical absorbance capacity (ORAC) in tissue}

Triglycerides were extracted from the LV using 5\% NP-40 according to the manufacturer's instruction (BDH Laboratory, Poole, Dorset, UK). After boiling and cooling to room temperature and centrifugation, the supernate was used for triglyceride assay (Fortress Diagnostics, Antrim, Northern Ireland, UK).

Total antioxidant capacity was analyzed by the ORAC assay as described by Hsieh et al. [13]. The assay measured fluorescence decay due to the breakdown of 2,2'-azobis (2-methylpropionamidine) dihydrochloride (AAPH). The antioxidant trolox served as a standard to inhibit the loss of fluorescence. Tissue lysate was mixed with $\beta$-phycoerythrin (PE). To begin the assay, fluorescence measurements (excitation: $515 \mathrm{~nm}$, emission: $577 \mathrm{~nm}$ ) were taken to determine the background signal. Then, AAPH was added and fluorescence was measured every 5 min until the value was below 100. Results were calculated using the differences of areas under the PE decay curves between the blank and each sample. The final results (ORAC values) were calculated by linear regression of the areas vs. sample concentration and expressed as $\mu$ mole trolox equivalents (TE) per $\mu \mathrm{g}$ of tissue protein $(\mu \mathrm{mol} \mathrm{TE} / \mu \mathrm{g})$.

\subsection{Measurements of oxidative stress}

Thiobarbituric acid reactive substances (TBARS) were measured as the marker for oxidative stress [11]. A left ventricular sample was homogenized in RIPA lysis buffer (Santa Cruz Biotechnology, Santa Cruz, CA, USA) and centrifuged at $14,000 \times g$ for $10 \mathrm{~min}$ at $4^{\circ} \mathrm{C}$. The supernate was mixed with thiobarbituric acid solution, and then mixed with trichloroacetic acid- $\mathrm{HCl}$ reagent. The mixture was boiled, and then cooled to room temperature and centrifuged at $5000 \times \mathrm{g}$ for $3 \mathrm{~min}$ at $4{ }^{\circ} \mathrm{C}$. The absorbance of the supernate was measured at $535 \mathrm{~nm}$ using microplate assays (BioTek, Synergy H1, Ashton Vale, BRS, USA) [11].

\subsection{Quantitative real-time polymerase chain reaction}

Total RNA was extracted from LV using the TRI reagent (Applied Biosystem, Grand Island, NY, USA) [14]. Two $\mu$ g of total RNA was reverse transcripted into cDNA. Then each cDNA was amplified using the SYBR Fast Master Mix (KAPA Biosystem, Woburn, MA, USA) and the StepOnePlus ${ }^{\mathrm{TM}}$ Real-time PCR System (Applied Biosystem, Grand Island, NY, USA). All primer sequences used and annealing temperatures are listed in Table 1 . The relative expression levels were calculated according to the formula $2^{-\triangle \mathrm{CT}}$ and normalized using the expression of $\beta$-actin in the same sample [15]. 
Table 1

Primer sequences of target genes.

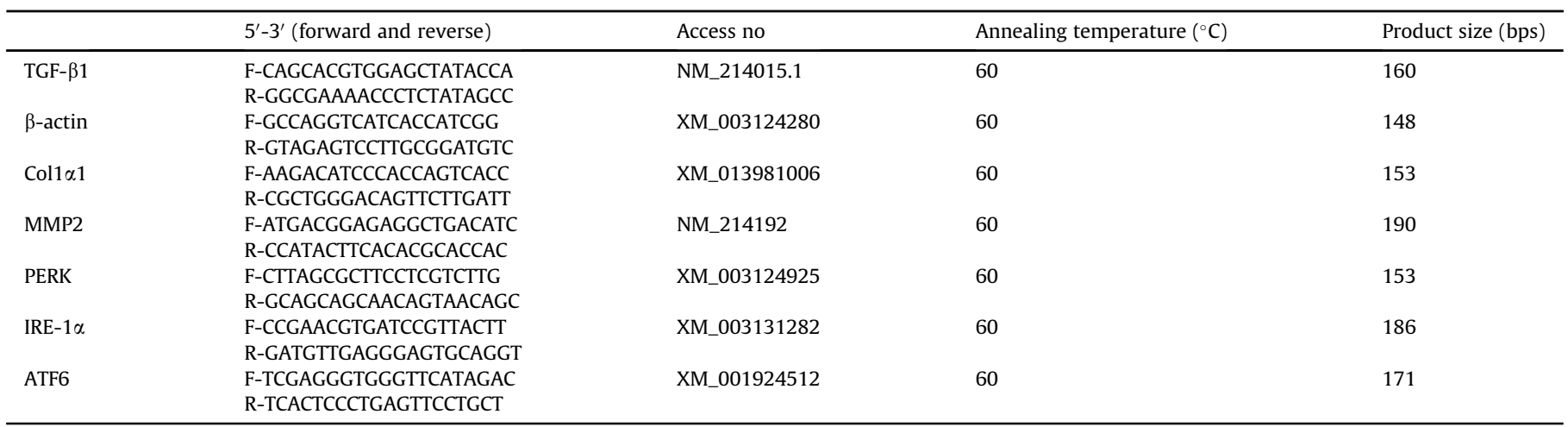

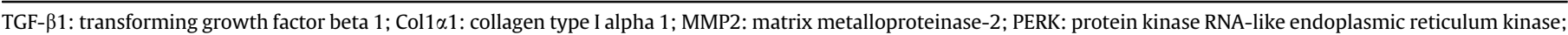
IRE-1 $\alpha$ : inositol-requiring enzyme 1 alpha; ATF6: activating transcription factor 6.

\subsection{Cytokine array}

Plasma cytokine profiles were analyzed using the Mouse Cytokine Array 3 (AAM-CYT-3) (RayBiotech, Norcross, GA, USA) according to the manufacturer's instructions. For the MetS and also for the $\mathrm{MHO}$ pigs $4 \mathrm{C}$ ( 2 barrows and 2 gilts) and $5 \mathrm{O}$ ( 2 barrows and 3 gilts) pigs were sampled. After blocking the membrane, $1 \mathrm{~mL}$ of porcine plasma was placed onto the membrane for incubation and then the membrane was washed. The membrane was then incubated with biotinylated antibody cocktails. After washing, the membrane was incubated with HRP-Streptavidin and then washed. The signals from immunoblotting were visualized and quantified using QUANTITY ONE (Bio-Rad, Hercules, CA, USA).

To distinguish the relationships among the treatments, unsupervised analyses of the array data were conducted using the $\mathrm{R}$ package (version 3.1.3). To observe the separation among groups, principle component analysis (PCA) was implemented using the R package PCA3D (version 0.8) [16]. The proportion of variance was also calculated.

\subsection{Western blotting}

Left ventricular samples were homogenized in RIPA lysis buffer (Santa Cruz Biotechnology) [17]. Protein concentrations were determined using Bradford assay reagents (B6916, Sigma, St. Louis, MO, USA). Tissue homogenate $(20 \mu \mathrm{g})$ was loaded onto a SDS-PAGE gel for electrophoresis and then transferred to a PVDF membrane (Bio-Rad). The membrane was then incubated with primary antibodies against 5' AMP-activated protein kinase (AMPK) (\#2532), microtubule-associated protein light chain 3 (LC3) (\#2775), p62 (\#5114), glucose related protein (Grp94) (ap4899a) (Abgent, San Diego, CA, USA), C/EBP homologous protein (CHOP) (\#5554), caspase 12 (\#2202), poly (ADP-ribose) polymerase (PARP) (\#9542), BCL2/adenovirus E1B $19 \mathrm{kDa}$ protein-interacting protein 3 (BNIP3) (\#3769) or GAPDH (\#2118) (Cell signaling Technology Inc. Hertfordshire, UK). After washing, the membrane was incubated with secondary antibody against goat or mouse IgG and the immunoblotting was visualized and quantified using QUANTITY ONE (BioRad).

\subsection{Statistical analysis}

Data were expressed as mean \pm standard error of the mean (SEM). The results were analyzed by one-way ANOVA followed by Fisher's test. Statistical analyses were performed using SAS (version
6.011; SAS Institute Inc, Cary, NC. USA). $P \leq 0.05$ was considered a significant difference.

\section{Results}

\subsection{The physiological parameters}

In accord with previous results [11], HFD feeding for 6 months induced MetS and MHO in obese Lee-Sung and Lanyu pigs, respectively. The current study investigated whether obesity induced cardiomyopathy in these two pig models. The present results showed that long-term HFD feeding increased the body mass index (BMI) in both MetS and MHO pigs (Fig. 1A).

Characteristics of the heart were displayed in Fig. 1B-I. Heavier heart weights are observed in both strains of obese pigs (Fig. 1B). In contrast, obese pigs had a smaller heart weight/BW than the control pigs (Fig. 1C). Obese MetS pigs (O-MetS) exhibited more cardiac lipid accumulation than the control MetS pigs (C-MetS), whereas CMHO and O-MHO pigs had similar cardiac lipid content (Fig. 1D). Collagen type I alpha 1 (COLA1), matrix metalloproteinase-2 (MMP2) and transforming growth factor beta 1 (TGF $\beta 1$ ) are the markers for cardiac fibrosis. The O-MetS pigs had an increase in the mRNA expression of TGF $\beta 1$, while O-MHO pigs had an increase in the expression of MMP2 and TGF $\beta 1$ as compared with C-MHO pigs (Fig. 1E and F). The histological results showed that in control pigs, cells were more compactly arranged in the LV, whereas in obese pigs, cells were arranged in a looser and distorted fashion (Fig. 1G). Interstitial and perivascular fibrosis developed in the myocardium of obese pigs.

Both Masson's trichrome and Sirius red staining were applied to quantify the degree of cardiac fibrosis. Figure $1 \mathrm{H}$ showed via Masson's trichrome staining, an elevated collagen content of the LV in both O-MetS and O-MHO pigs (Fig. 1H). Similar results were found in the Sirius red staining (Fig. 1I), i.e., obese pigs had higher levels of collagen than the control pigs. Taken together, our data demonstrated that cardiac fibrosis and hypertrophy were induced in both O-MetS and O-MHO pigs.

\subsection{Redox status in the $L V$}

The redox status was evaluated by total antioxidant capacity (measured as ORAC) and oxidative stress (measured as TBARS) (Fig. 2). Compared with C-MetS pigs, O-MetS pigs had a lower ORAC value in the LV, but no difference was found between the $\mathrm{C}$ - and $\mathrm{O}$ MHO pigs. Both obese MetS and MHO pigs exhibited an increase in 
A

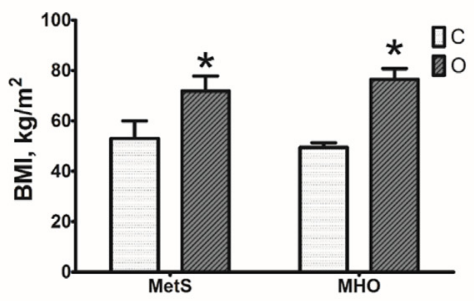

D

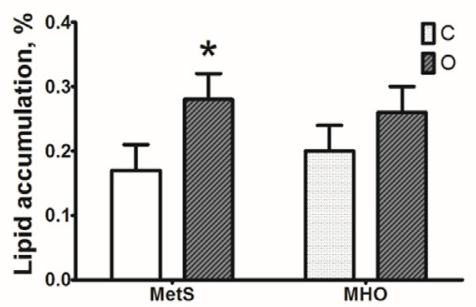

B

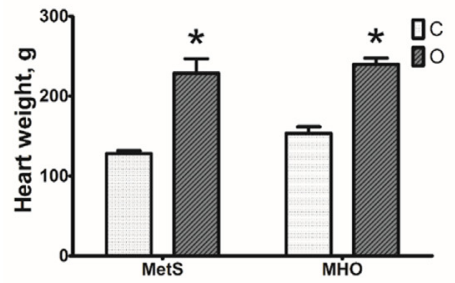

E

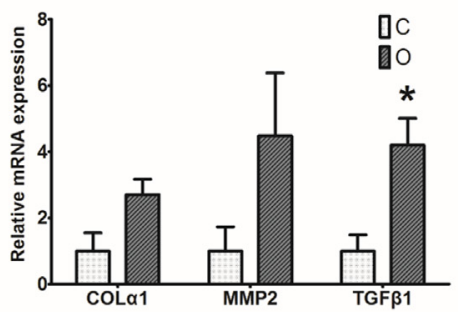

C

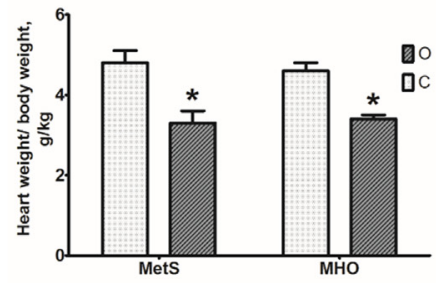

$\mathrm{F}$

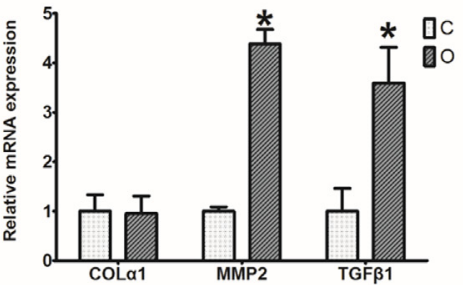

G

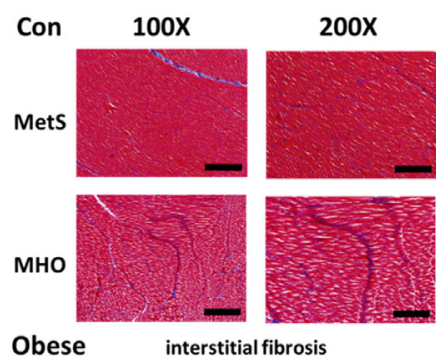

Obese interstitial fibrosis
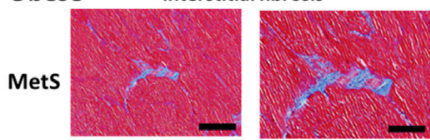

Мно
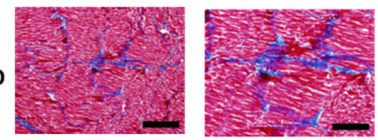

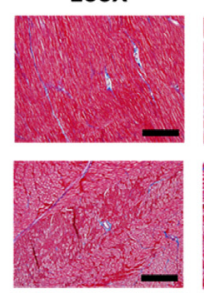

$200 x$
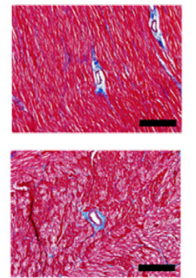

Perivascular fibrosis
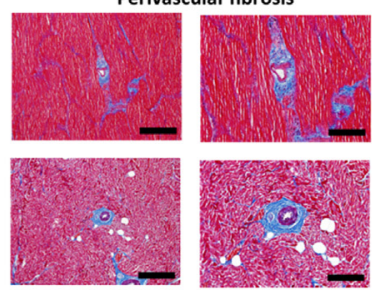

$\mathrm{H}$

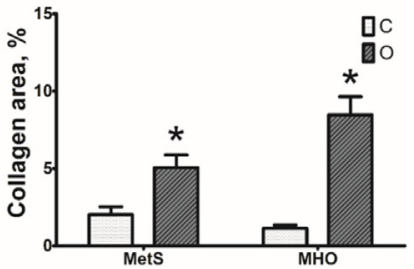

I

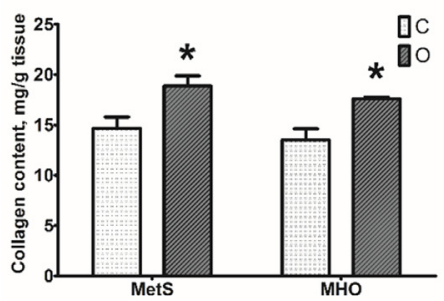

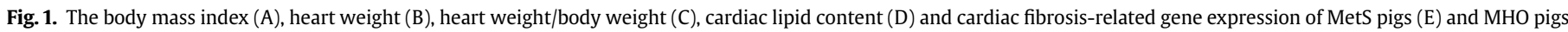

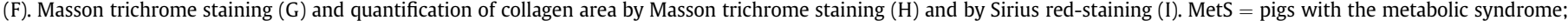

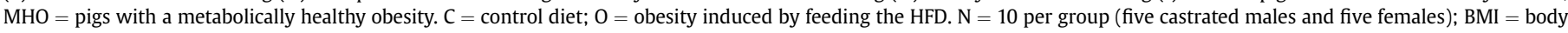

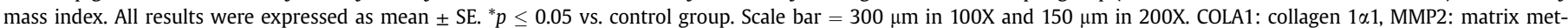

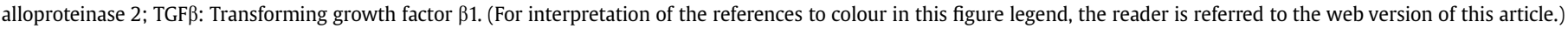

cardiac TBARS level. These data indicated that the HFD induced oxidative stress and decreased antioxidant defense in O-MetS pigs; in O-MHO pigs, HFD activated oxidative stress but did not change the antioxidant defense.

\subsection{Expression of gene and protein in the $L V$}

The protein quality control system (autophagy and ER stress) performs the essential function of maintaining cellular homeostasis. Activating transcription factor 6 (ATF6), PERK and inositol-requiring kinase/endoribonuclease $1 \alpha$ (IRE1 $\alpha$ ) are the major regulators for the signaling pathway of ER stress.
Compared with the C-MetS pigs, O-MetS pigs had a significant increase in the cardiac mRNA expression of PERK and ATF6 (Fig. 3A). The Grp94 is the marker for ubiquitin protein response (UPR), whereas CHOP and caspase 12 represent ER stress related apoptosis. The HFD upregulated the cardiac expression of Grp94, CHOP and pro-caspase 12 in O-MetS compared to C-MetS pigs (Fig. 3B). The AMPK, LC3II and p62 were monitored as markers for autophagy initiation, autophagosome accumulation and autophagy clearance, respectively. In the MetS pig model, the HFD activated AMPK activity, increased p62 expression and the ratio of LC3II to LC3I (Fig. 3C). Proteolytic cleavage of PARP by caspases is applied as the marker of apoptosis. Compared with 
A

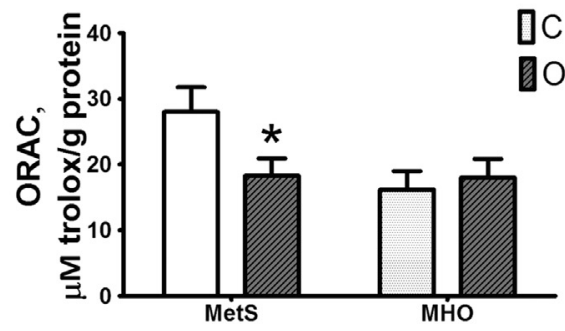

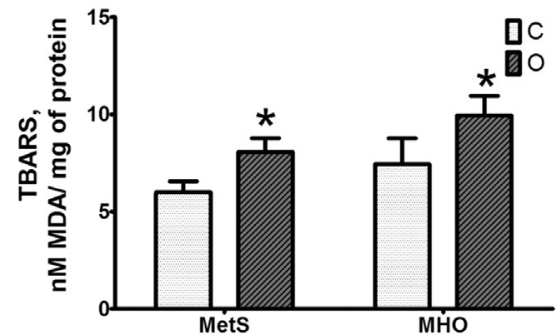

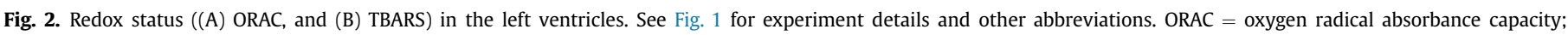
TBARS $=$ thiobarbituric acid reactive substances. $\mathrm{N}=10$. All results were expressed as mean $\pm \mathrm{SE} .{ }^{*} p \leq 0.05$ vs. control group.

A
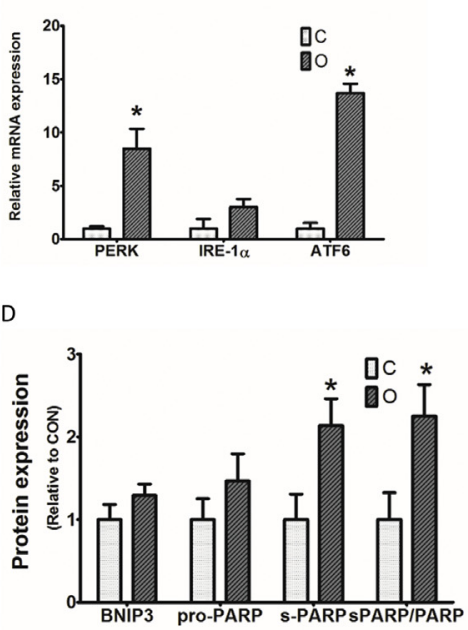

B

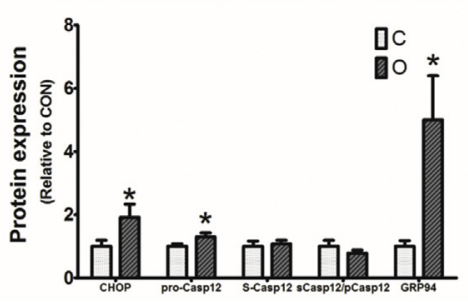

C

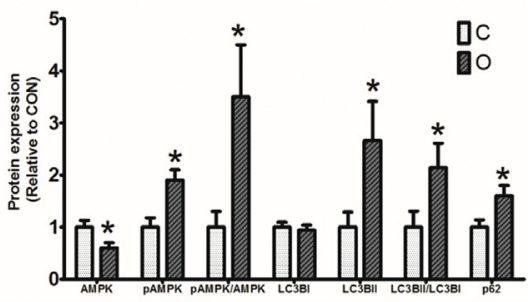

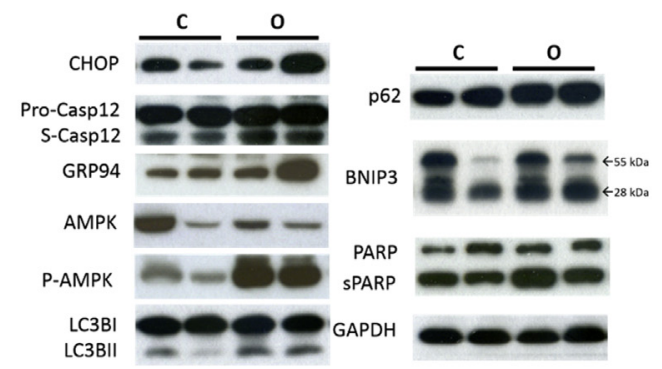
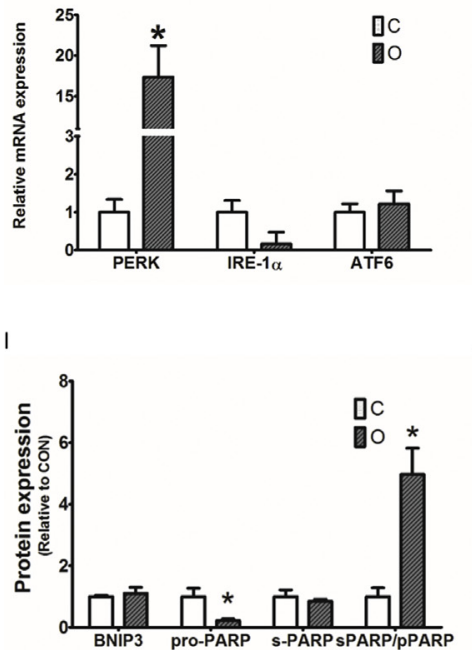

G

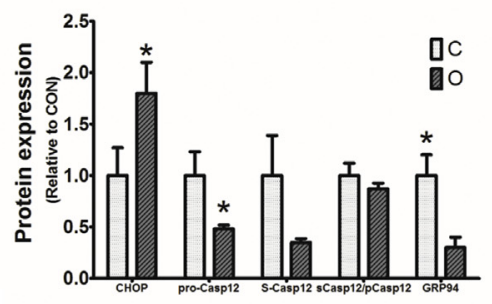

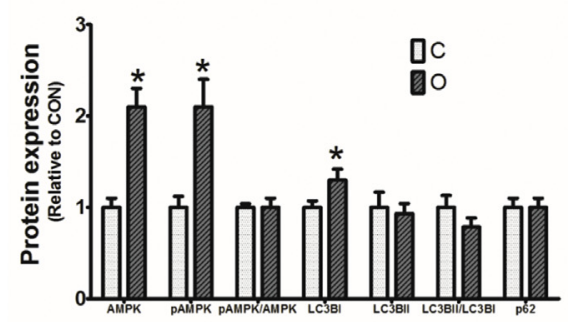

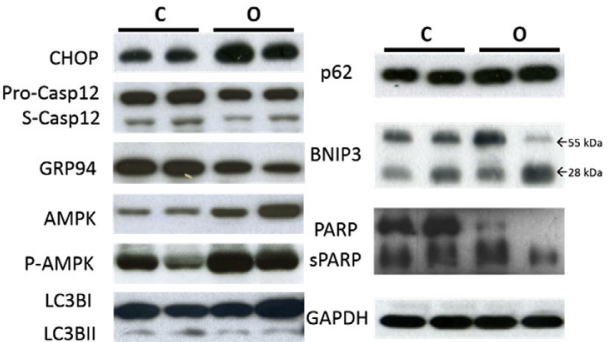

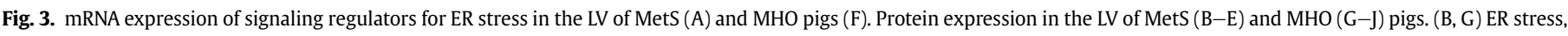

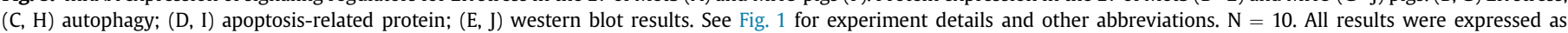

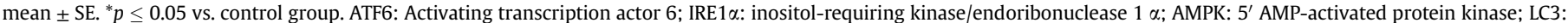

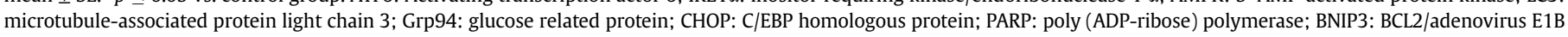
$19 \mathrm{kDa}$ protein-interacting protein 3. 
C-MetS pigs, O-MetS pigs had a higher cleaved PARP level in the LV (Fig. 3D).

A different cellular response to the HFD was observed in the MHO pig model (Fig. 3F-J). The HFD increased the mRNA expression of PERK in O-MHO pigs (Fig. 3F). Compared to C-MHO pigs, less Grp94 and pro-caspase 12 were expressed in O-MHO pigs; higher CHOP expression was found in O-MHO than C-MHO pigs (Fig. 3G). The HFD elevated cardiac expression of AMPK, phosphorylated AMPK, LC3I, however, HFD did not change the ratio of LC3II to LC3I and p62 expression (Fig. 3H). The ratio of cleaved PARP to PARP was increased by HFD (Fig. 3I). Taken together, in the MetS pigs, the HFD increased the UPR, induced PERK and ATF6-dependent signaling and caused ER stress-related apoptosis. A similar pattern was observed in the autophagic function, i.e., the HFD initiated autophagy, but failed to clear autophagosomes (Fig. 3C). In the MHO pigs, HFD inhibited UPR and caused ER stress-related apoptosis in a PERK-dependent manner. HFD activated functional autophagy, and did not cause excess autophagosome accumulation.

\subsection{Plasma cytokine profiles}

A total of 62 cytokines were assessed to investigate the plasma cytokine profile in pigs. The Principle Component Analysis was performed to distinguish the four groups. In the scatter plots, each point represents a chip and the color of the dot represents the type of treatment. Points that are close together within the plots have similar intensity values, and points that are far apart within the plots are dissimilar. As seen in Fig. 4A, O-MetS pigs were clearly separated from the other three groups, demonstrating that they were dissimilar to others. PCA separated the control pigs into 2 distinct populations (C-MetS and C-MHO). It is noticeable that some O-MHO pigs are close to C-MHO pigs. The proportion of variance show that PC1 (principle component 1) explained $72.8 \%$ among 18 samples (Fig. 4B). The PCA results demonstrated that O-
MetS pigs had a distinct cytokine profile from others, whereas the cytokine profile cannot be distinguished between $\mathrm{C}-\mathrm{MHO}$ and $\mathrm{O}$ MHO pigs.

Selected cytokine profiles were indicated in Fig. 4C and totally in Supplementary Tables 1 and 2. The C-MetS had 25 cytokines with greater concentration than in C-MHO pigs. Similar patterns were found between O-MHO and O-MetS pigs with 20 plasma cytokines being at greater concentration in O-MetS pigs than in OMHO pigs. Compared with the control pigs the HFD induced an extensive and greater inflammatory response in O-MetS pigs, whereas the HFD caused a milder inflammation in O-MHO pigs (26 and 10 cytokines elevated for MetS and MHO, respectively). Figure $4 \mathrm{C}$ shows that the plasma cytokines were significantly upregulated by the HFD in pigs. Additionally, O-MetS pigs had greater levels of these cytokines than O-MHO pigs.

\section{Discussion}

We previously established two obese animal models, one MetS and one MHO, for the study of metabolic related diseases [11]. The present study demonstrated that cardiomyopathy was exhibited in both MetS and MHO obese pigs. Interestingly, distinct cellular responses and plasma cytokine profiles were observed in these two pig models, indicating a different pathogenic mechanism involved in MetS- and MHO-related cardiomyopathy.

There is a stronger risk of heart failure associated with longterm obesity, regardless of metabolic status, compared with normal-weight and metabolically healthy participants [7]. Similar results were confirmed in our porcine study. Cardiomyopathy was induced in both $\mathrm{MHO}$ and MetS obese pigs. However, the exact mechanisms involved in MHO-related cardiac damage are not well defined.

The present study showed that interstitial and perivascular fibrosis developed in the myocardium of O-MHO pigs. Clinical
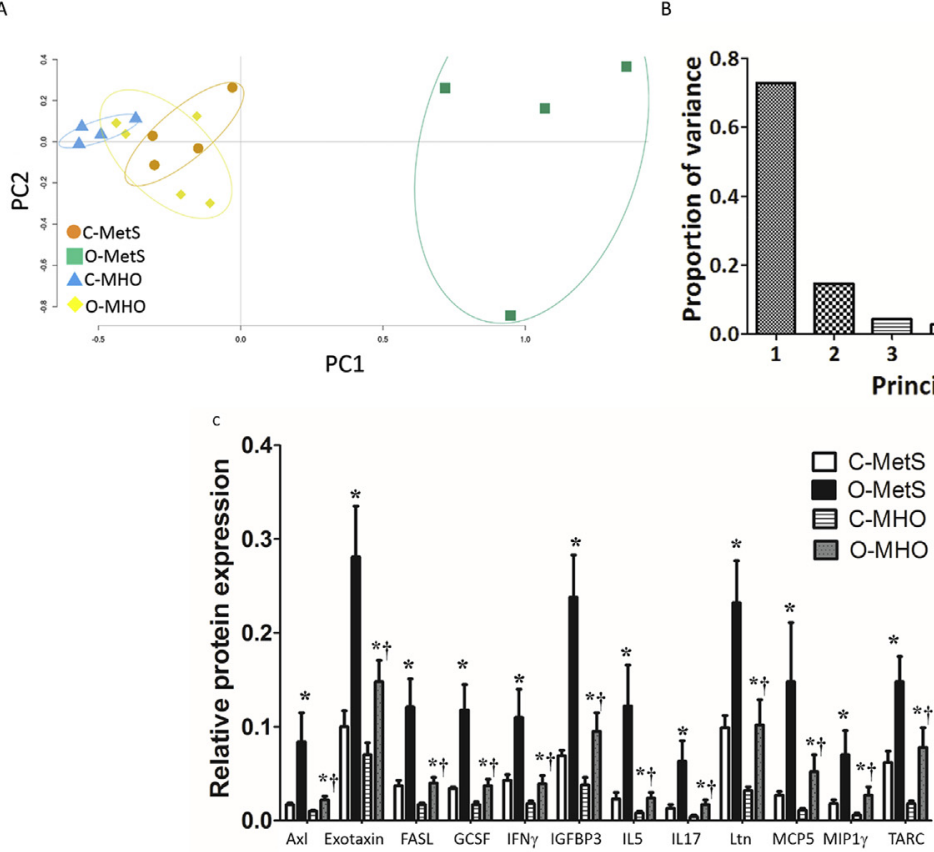

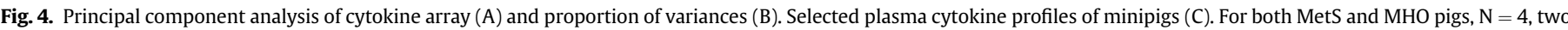

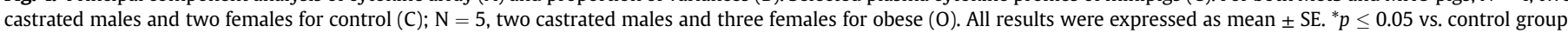

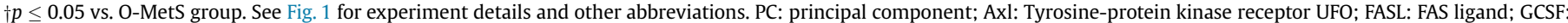

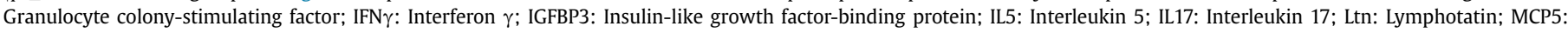
monocyte chemotactic protein 5; MIP1 $\gamma$ : Macrophage inflammatory protein $1 \gamma$; TARC: Thymus- and activation-regulated chemokine. 
studies demonstrate considerable association between obesity and cardiac fibrosis; potential mechanisms contributing to cardiac fibrosis include hemodynamic factors, neuro-humoral substances, metabolic dysregulation, inflammation and oxidative stress [18,19]. Nevertheless, we previously found that neither metabolic dysfunction (hyperglycemia, insulin resistance and dyslipidemia) nor hypertension was induced in O-MHO pigs [11]. These results indicate that the pathogenic mechanisms of cardiac fibrosis in the O-MHO heart do not include metabolic abnormalities or pressure overload. The present study suggests that structural changes induced by obesity and oxidative stress are the main contributors to the pathogenesis of O-MHO-associated cardiac fibrosis.

We found that ER stress (CHOP dependent), but not autophagy was associated with cardiac fibrosis in the O-MHO heart. The cellular protein quality system includes autophagy and the functional ER. When exposed to cellular stresses, adaptive autophagy is activated to form autophagosomes and degradation of the damaged proteins in a lysosome system [20]. Also, the UPR in the ER is activated to restore cellular homeostasis [21]. Failure to maintain the basic functions of the ER and autophagy causes excessive autophagosome accumulation and ER stress, eventually leading to cell apoptosis. The ER stress is a pro-fibrotic stimulus [22] and attenuating ER stress decreases cardiac fibrosis [23]. Accordingly, we postulated that the HFD induced oxidative stress to further elevate ER stress, thus causing cardiomyocyte apoptosis and cardiac fibrosis in $\mathrm{O}-\mathrm{MHO}$ pigs.

To investigate the inflammatory state of the animals, we used an inflammatory cytokine array. Remarkably, only a small scale and mild inflammation was found in the O-MHO pigs, suggesting a minor effect on the progression of this cardiomyopathy. However, the 10 elevated cytokines in the blood of O-MHO pigs (Fig. 4C), implied their potential role in the pathogenesis of the O-MHOrelated cardiomyopathy.

Among the cytokines upregulated by our HFD, eotaxin is the one demonstrated as a contributor to cardiac fibrosis [24]. Eotaxin is an important inflammatory chemokine in eosinophil chemotaxis. Eotaxin expression levels increase with adipocyte differentiation [25], whereas its levels decrease in visceral adipose tissues when HFD-fed mice undergo weight loss [25]. In addition, eotaxin participates in cardiac fibrogenesis by triggering mast cell infiltration of the heart [24]. The O-MHO pigs exhibited a higher plasma level of eotaxin than C-MHO pigs, suggesting its potential role in the development of cardiac fibrosis. Although there is no literature indicating that the other 9 cytokines participate in cardiac fibrosis, roles in fibrogenesis of liver (Axl) and lung (IL5, IL17, and TARC), suggest that they may be involved in cardiac fibrosis and apoptosis [26-29].

Taken together, our data suggested that oxidative stress and ER stress participated in the pathogenesis of O-MHO-associated cardiomyopathy and that inflammation may be a minor contributor.

One interesting issue concerning inflammation was noticed. The HFD elevated the plasma level of granulocyte colony-stimulating factor (GCSF), but did not change the level of granulocyte macrophage-colony stimulating factor (GMCSF) in the obese pigs. Both GMCSF and GCSF are hematopoietic cytokines which stimulate granulocyte proliferation and mobilize bone marrow stem cells [30]. However, a different regulation pattern is noticed during the development of heart failure. The GCSF reduces myocardial injury and improves cardiac function in rats with acute and chronic myocardial infarction [31,32] and is regarded as a potential cardiac repair therapy for subjects with myocardial infarction [30]. The GMCSF is able to enhance collateral blood flow of patients with chronic myocardial infarction [33]; however, plasma levels of GMCSF in patients with heart failure are highly associated with LV dysfunction [34]. Moreover, GMCSF exacerbates early post- infarction LV remodeling [30,35]. Our results indicated a different regulation pattern between pigs and rodents or humans. The role of hematopoietic cytokines in pigs with obesity-related cardiomyopathy remains unclear and needs further investigation.

A recent study shows a high-fat, high-sucrose diet (HFHSD)induced cardiomyopathy minipig model similar to our O-MetS pig model. Xia et al. fed a HFHSD to Bama minipigs to induce obesity [36]. They found that these obese pigs developed the metabolic syndrome, and showed cardiac steatosis and hypertrophy. The transcriptomic profiling of heart indicated that immune responses and inflammatory cytokine production are significantly upregulated in the HFHSD miniature pigs. Our results were similar to those of Xia et al. with considerable systemic inflammation and cardiac fibrosis. However, the feeding period to induce obesity in our OMetS model was significantly shorter (6 vs. 23 months), indicating our O-MetS pigs are a preferable animal model for studying MetSrelated cardiomyopathy.

To summarize, our study demonstrated a different cellular response and plasma cytokine profiles for obese MetS and obese MHO-related cardiomyopathies. The HFD induced structural changes/hypertrophy in the heart and increased the oxidative stress and ER stress (CHOP-dependent), all of which may partially contribute to the progression of O-MHO-related cardiomyopathy. For the O-MetS pigs, cardiomyopathy, inflammation, disrupted cellular homeostasis (autophagy and ER stress), intensive oxidative stress and LV fat deposition were involved in the pathological mechanism. Inhibiting ER stress and oxidative stress could be a potential therapeutic approach for the MHO cardiomyopathy, but differences between humans and animal models must be considered.

\section{Conflict of interest}

None declared.

\section{Acknowledgments}

This work was supported by the Research Grant MOST 1042313-B-002-038-MY3 (from Ministry of Science and Technology) and NTU-CESRP-104R7615-3 (from National Taiwan University). The authors are grateful to Dr. Yan-Nian Jiang for his statistical assistance, and Mr. Chi-Hui Chen for his technical assistance.

\section{Appendix A. Supplementary data}

Supplementary data related to this article can be found at http:// dx.doi.org/10.1016/j.clnu.2016.06.002.

\section{References}

[1] Huang KC. Obesity and its related diseases in Taiwan. Obes Rev 2008:9(Suppl. 1):32-4.

[2] Mottillo S, Filion KB, Genest J, Joseph L, Pilote L, Poirier P, et al. The metabolic syndrome and cardiovascular risk a systematic review and meta-analysis. J Am Coll Cardiol 2010;56:1113-32.

[3] Despres JP, Lemieux I, Bergeron J, Pibarot P, Mathieu P, Larose E, et al. Abdominal obesity and the metabolic syndrome: contribution to global cardiometabolic risk. Arterioscler Thromb Vasc 2008:28:1039-49.

[4] Wildman RP, Muntner P, Reynolds K, McGinn AP, Rajpathak S, Wylie-Rosett J, et al. The obese without cardiometabolic risk factor clustering and the normal weight with cardiometabolic risk factor clustering: prevalence and correlates of 2 phenotypes among the US population (NHANES 1999-2004). Arch Intern Med 2008;168:1617-24

[5] Appleton SL, Seaborn CJ, Visvanathan R, Hill CL, Gill TK, Taylor AW, et al. Diabetes and cardiovascular disease outcomes in the metabolically healthy obese phenotype: a cohort study. Diabetes Care 2013:36:2388-94.

[6] Hinnouho GM, Czernichow S, Dugravot A, Nabi H, Brunner EJ, Kivimaki M, et al. Metabolically healthy obesity and the risk of cardiovascular disease 
and type 2 diabetes: the Whitehall II cohort study. Eur Heart J 2015;36: 551-9.

[7] Morkedal B, Vatten LJ, Romundstad PR, Laugsand LE, Janszky I. Risk of myocardial infarction and heart failure among metabolically healthy but obese individuals. J Am Coll Cardiol 2014;63:1071-8.

[8] Lavie CJ, Alpert MA, Arena R, Mehra MR, Milani RV, Ventura HO. Impact of obesity and the obesity paradox on prevalence and prognosis in heart failure. JACC Heart Fail 2013;1:93-102.

[9] Hsu HC, Liu CH, Tsai YC, Li SJ, Chen CY, Chu CH, et al. Time-dependent cellular response in the liver and heart in a dietary-induced obese mouse model: the potential role of ER stress and autophagy. Eur J Nutr 2015. http://dx.doi.org/ 10.1007/s00394-015-1017-8.

[10] Hsu HC, Chen CY, Li SJ, Lee BC, Chen MF. High-fat diet consumption induces cardiomyocyte apoptosis via autophagy inhibition. Eur J Nutr 2015. http:// dx.doi.org/10.1007/s00394-015-1034-7.

[11] Li SJ, Liu CH, Chang CW, Chu HP, Chen KJ, Mersmann HJ, et al. Development of a dietary-induced metabolic syndrome model using miniature pigs involvement of AMPK and SIRT1. Eur J Clin Investig 2015;45:70-80.

[12] Baxter EM, Jarvis S, D'Eath RB, Ross DW, Robson SK, Farish M, et al. Investigating the behavioural and physiological indicators of neonatal survival in pigs. Theriogenology 2008;69:773-83.

[13] Hsieh YH, Wang HT, Hsu JT, Chen CY. Albusin B, mass-produced by the Saccharomyces cerevisiae suppression system, enhances lipid utilisation and antioxidant capacity in mice. J Sci Food Agric 2013;93:2758-64.

[14] Li SJ, Ding ST, Mersmann HJ, Chu CH, Hsu CD, Chen CY. A nutritional nonalcoholic steatohepatitis minipig model. J Nutr Biochem 2016;28:51-60.

[15] Schmittgen TD, Livak KJ. Analyzing real-time PCR data by the comparative CT method. Nat Protoc 2008;3:1101-8.

[16] Weiner J. pca3d: three dimensional PCA plots. R package version 0.8. 2015. https://CRAN.R-project.org/package=pca3d.

[17] Chen CY, Hsu HC, Lee BC, Lin HJ, Chen YH, Huang HC, et al. Exercise training improves cardiac function in infarcted rabbits: involvement of autophagic function and fatty acid utilization. Eur J Heart Fail 2010;12:323-30.

[18] Kong P, Christia P, Frangogiannis NG. The pathogenesis of cardiac fibrosis. Cell Mol Life Sci 2014;71:549-74.

[19] Cavalera M, Wang J, Frangogiannis NG. Obesity, metabolic dysfunction, and cardiac fibrosis: pathophysiological pathways, molecular mechanisms, and therapeutic opportunities. Transl Res 2014;164:323-35.

[20] Ren SY, Xu X. Role of autophagy in metabolic syndrome-associated heart disease. Biochim Biophys Acta 2015;1852:225-31.

[21] Minamino T, Kitakaze M. ER stress in cardiovascular disease. J Mol Cell Cardiol 2010;48:1105-10.

[22] Tanjore H, Lawson WE, Blackwell TS. Endoplasmic reticulum stress as a profibrotic stimulus. BBA-Mol Basis Dis 2013;1832:940-7.

[23] Ayala P, Montenegro J, Vivar R, Letelier A, Urroz PA, Copaja M, et al. Attenuation of endoplasmic reticulum stress using the chemical chaperone 4phenylbutyric acid prevents cardiac fibrosis induced by isoproterenol. Exp Mol Pathol 2012;92:97-104.
[24] Zweifel M, Matozan K, Dahinden C, Schaffner T, Mohacsi P. Eotaxin/CCL11 levels correlate with myocardial fibrosis and mast cell density in native and transplanted rat hearts. Transplant Proc 2010;42:2763-6.

[25] Kim HJ, Kim CH, Lee DH, Han MW, Kim MY, Ju JH, et al. Expression of eotaxin in 3T3-L1 adipocytes and the effects of weight loss in high-fat diet induced obese mice. Nutr Res Pract 2011;5:11-9.

[26] Fourcot A, Couchie D, Chobert MN, Zafrani ES, Mavier P, Laperche Y, et al. Gas6 deficiency prevents liver inflammation, steatohepatitis, and fibrosis in mice. Am J Physiol Gastrointest Liver Physiol 2011:300:G1043-53.

[27] Yogo Y, Fujishima S, Inoue T, Saito F, Shiomi T, Yamaguchi K, et al. Macrophage derived chemokine (CCL22), thymus and activation-regulated chemokine (CCL17), and CCR4 in idiopathic pulmonary fibrosis. Respir Res 2009;10:80.

[28] Emad A, Emad Y. Relationship between eosinophilia and levels of chemokines (CCL5 and CCL11) and IL-5 in bronchoalveolar lavage fluid of patients with mustard gas-induced pulmonary fibrosis. J Clin Immunol 2008;28: 298-305.

[29] Conte E, Iemmolo M, Fagone E, Gili E, Fruciano M, Genovese T, et al. Thymosin beta 4 reduces IL-17-producing cells and IL-17 expression, and protects lungs from damage in bleomycin-treated mice. Immunobiology 2014:219:425-31.

[30] Sanganalmath SK, Abdel-Latif A, Bolli R, Xuan YT, Dawn B. Hematopoietic cytokines for cardiac repair: mobilization of bone marrow cells and beyond. Basic Res Cardiol 2011:106:709-33.

[31] Baldo MP, Davel AP, Nicoletti-Carvalho JE, Bordin S, Rossoni LV, Mill JG. Granulocyte colony-stimulating factor reduces mortality by suppressing ventricular arrhythmias in acute phase of myocardial infarction in rats. J Cardiovasc Pharmacol 2008:52:375-80.

[32] Baldo MP, Davel AP, Damas-Souza DM, Nicoletti-Carvalho JE, Bordin S, Carvalho HF, et al. The antiapoptotic effect of granulocyte colony-stimulating factor reduces infarct size and prevents heart failure development in rats. Cell Physiol Biochem 2011;28:33-40.

[33] Seiler C, Pohl T, Wustmann K, Hutter D, Nicolet PA, Windecker S, et al. Promotion of collateral growth by granulocyte-macrophage colony-stimulating factor in patients with coronary artery disease: a randomized, double-blind, placebo-controlled study. Circulation 2001;104:2012-7.

[34] Parissis JT, Adamopoulos S, Venetsanou K, Kostakis G, Rigas A, Karas SM, et al Plasma profiles of circulating granulocyte-macrophage colony-stimulating factor and soluble cellular adhesion molecules in acute myocardial infarction. Contribution to post-infarction left ventricular dysfunction. Eur Cytokine Netw 2004;15:139-44.

[35] Naito K, Anzai T, Sugano Y, Maekawa Y, Kohno T, Yoshikawa T, et al. Differential effects of GM-CSF and G-CSF on infiltration of dendritic cells during early left ventricular remodeling after myocardial infarction. J Immuno 2008; 181:5691-701.

[36] Xia J, Zhang Y, Xin L, Kong S, Chen Y, Yang S, et al. Global transcriptomic profiling of cardiac hypertrophy and fatty heart induced by long-term highenergy diet in Bama miniature pigs. PLoS One 2015;10:e0132420. 